\title{
New evidence for "closure" in perception
}

\author{
BARBARA GILLAM \\ State University of New York, State College of Optometry \\ 122 East 25 Street, New York, New York 10010
}

\begin{abstract}
A new criterion of perceptual grouping (Gillam, 1972), namely, that contours appear to reverse together under conditions of depth ambiguity, was used to test for the reality of "closure" and explore the parameters determining its degree. It was found that contours which would form a closed space but for a linear gap were responded to as a unit to a degree which decreased monotonically with gap size. Neither "proximal" nor "distal" gap was found to be the sole determinant of closure. Several levels of processing appear to be involved.
\end{abstract}

The Gestalt psychologists (Koffka, 1935) stated that closed organizations of lines are preferred to open organizations, and that figures with parts removed tend to be perceived as complete figures if the remaining parts enclose or surround space. It is not always easy to specify precisely what this statement means. The necessary stimulus and/or the criterion response are often not defined and the very term "closure" has come under fire (Schoenfeld, 1941).

One well-known criterion of closure is omission of gaps in the subject's reproductions of stimuli after brief exposure or after a delay from stimulus offset. However, Holmes (1968) showed in an extensive review that in most memory studies exaggeration of gap size rather than closure occurred. A second well-known criterion involves the recognition of complex figures obliterated in a piecemeal fashion (see Street Gestalt Completion Test, Street, 1931). However, this criterion requires many gaps to be "closed" before the final response can occur, which makes it a poor tool to separate and investigate underlying processes. The concern of the present paper is therefore with simpler figures, such as the common textbook example shown in Figure 1 (after Heidbreder, 1933). Here "closure" is said to occur although the gaps are clearly visible, subjective contours are not present, and no meaningful object is obscured by the gaps. In what sense, if any, can a specific gap in such a figure be said to be perceptually closed? The lack of any operational definition of closure in discussions of such simple figures is perhaps a tribute to the phenomenon, but does not allow for very satisfying scientific investigation. For example, in Bobbit's (1942) study, verbal responses were used to find the threshold gap size for perceived "oneness" of triangular figures. However, although the work was carefully done, it is not clear from either

This work was supported by PHS Grant 5-R01-EY-00391 from the National Eye Institute and Grant B043254 from the National Science Foundation.

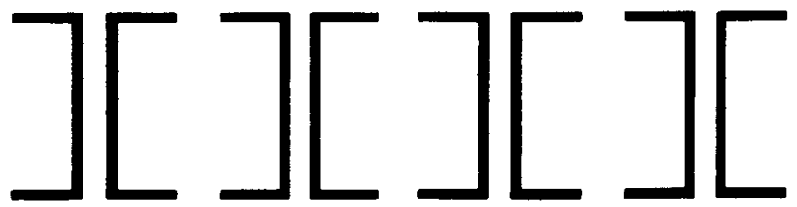

Figure 1. Standard textbook figure to illustrate closure. The lines are said to be organized according to the principle of closure rather than the principle of proximity.

the text or instructions just what the terms "closure" or "oneness" meant to either the subject or the experimenter.

The present investigation of "closure" arose out of a broader investigation of perceived coherence or "grouping" (Gillam, 1972). A novel criterion was developed in that paper for grouping, namely, that contours display simultaneous reversal in depth under conditions of depth ambiguity. Oblique lines of opposite orientations appear to reverse independently to a considerable degree, whereas parallel lines almost always reverse together. By adding closure-inducing lines to contours that tend to reverse independently, one can create figures in which closure would be expected. If closure does occur, it would be revealed by a shift from independent reversal to simultaneous reversal in depth. The figures used and the technique for measuring the degree of grouping or closure are described in detail below. Closure was indeed found to occur to a degree which varied with gap size and with the retinal size of the entire configuration.

\section{METHOD}

To achieve depth ambiguity, Gillam's (1972) technique for measuring perceptual grouping used the kinetic depth effect (Wallach \& O'Connell, 1953) for projections of rotating oblique contours. Parallel projections of such rotating lines are ambiguous with respect to direction of motion, and reversals are frequent. Two such lines will not always reverse together, but will often appear to rotate in opposite directions (one clockwise and the other counterclockwise) (Gillam, 1972). This is so even for the oblique lines of Figure $2 b$ which are joined on one side. It is not surprising 

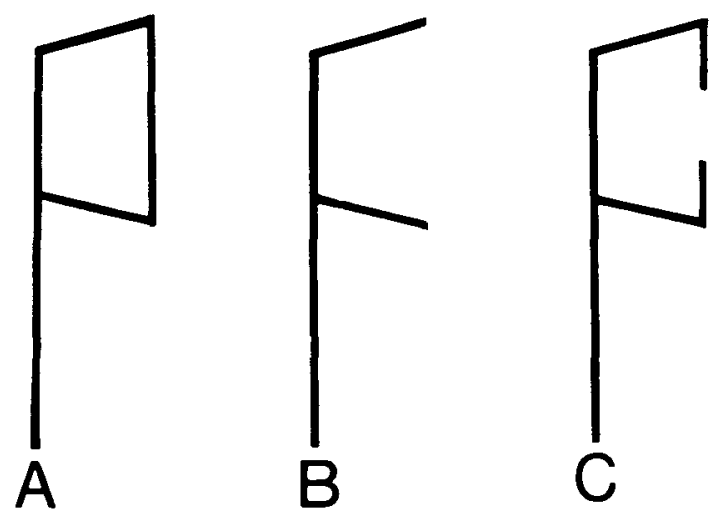

Figure 2. (A) A closed figure. (B) An open figure. (C) A figure of the type used in Experiments $I$ and II.

that if both sides are joined, as in Figure $2 a$. the oblique lines always appear to rotate in the same direction as each other and reverse together, but this is itself a powertul demonstration that closed tigures are organized as a unit. In the present experiment. vertical appendages were attached to the upper and lower obliques of Figure $2 a$. creating figures like $2 b$ except for a central gap in the longer vertical side. Appendage length, and therefore gap, varied from figure to figure. An example is shown in Figure 2c. The proportion of time that the upper and lower oblique lines of the figure with their vertical appendages appeared to be moving in opposite directions was the quantitative measure of their independence.

\section{EXPERIMENT I}

\section{Apparatus and Procedure}

The apparatus and procedure are described elsewhere (Gillam, 1972). Brietly, subjects were required to view parallel projections of wire tigures like Figure $2 c$ (which is drawn to scale) rotating on a vertical axis at $12 \mathrm{sec}$ revolution for 10 revolutions. Binocular vision was used, since it did not appear to reduce the kinetic depth effect and was more confortable for the subject. The distance between the upper and lower oblique lines along the vertical axis was 1 deg of visual angle at a vieuing distance of $1.400 \mathrm{~mm}$. The gap was always centered and gap size varied from $1.52 \mathrm{~mm}$ $(3.7 \mathrm{~min}$ of visual angle) to $32 \mathrm{~mm}(78.9 \mathrm{~min})$ in a side length of $39 \mathrm{~mm}(96.1 \mathrm{~min})$. For each figure, the subject was asked to fixate the vertical line midway between the obliques and to press and hold down a switch for the duration of time that the upper and lower oblique lines appeared to rotate in opposite directions (fragmentation time). This time was cumulated on a clock for the 10 revolutions for each figure. The total number of presses for each figure was also recorded. Ten subjects were used, each doing all conditions in a random order.

\section{Results}

The results for the 10 subjects are shown in Figure 3. Fragmentation time was a monotonic function of gap size. In other words, closure is an inserse monotonic function of gap size.

\section{CONTROL EXPERIMENT}

An assumption underlying Experiment $I$ is that the apparent motion of a single oblique line (see Figure 4) of any of the figures used will not vary with the length of its vertical appendage. lf, for example, the tendency of an observer to see the appendage pointing towards hin were to increase with appendage length, then the present results could be accounted for by addition of the independent motions of the upper and lower oblique lines without postulating an interaction. Although this did not seem a likely explanation of the results, a control experiment was conducted to exclude it. In the control experiment, apparent motion patterns were measured for two halt-figures (upper oblique only), one with a short vertical appendage and one with a long vertical appendage. A substantial difference in their motion patterns might cast doubt on the interpretation of Experiment $I$ as closure of a gap.

\section{Method}

The figures used were those from Experiment 1 with the next to greatest gap (A) and the next to smallest gap (B) (see Figure 3 for values). The lower half of the silhouette of each tigure was blocked out by a screen so that the figures appeared as in Figure 4. Ten new subjects riewed each of the figures rotating tive times each in the order ABBA or BAAB. Orders were assigned to the subjects alternately. The subject was told, "You will see an oblique line with a vertical hook moving on a screen. The tw 0 -dimensional motion on the screen can be regarded as having four phases repeating themselves - hook on the right moving out from the axis, hook on the right moving into the axis, hook on the left moving out from the axis, hook on the left moving in to the axis. (This was illustrated.) I want you to report during each of these four phases whether the hook appears to be moving towards or away from you in depth. You will call out "towards" or "away" during each phase." The subjects appeared to have no difticulty with this task. The sinusoidal motion in the projection of a rotating oblique line always appears overwhelmingly three-dimensional.

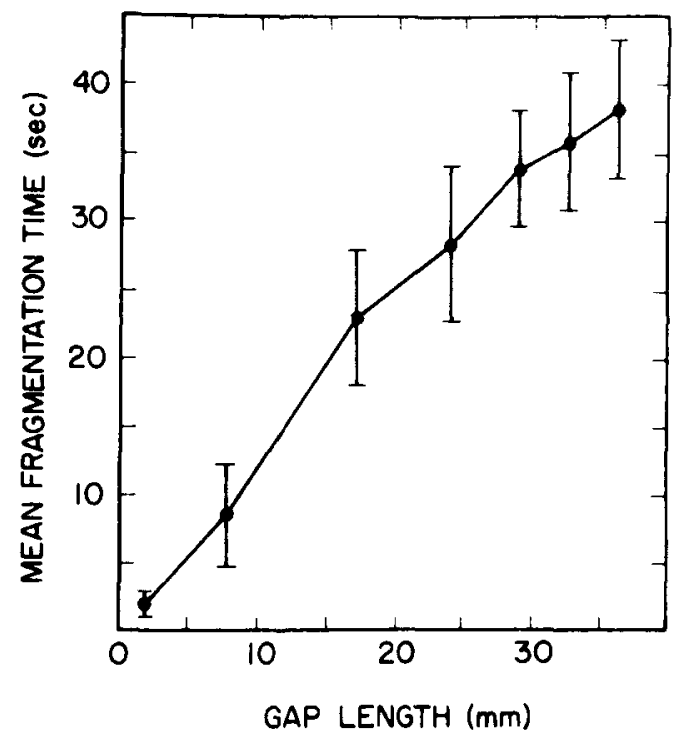

Figure 3. Mean fragmentation time as a function of gap size. Standard errors are shown. 

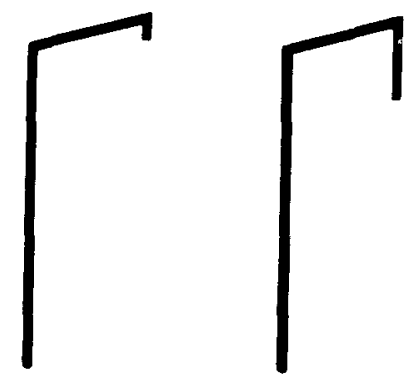

Figure 4. The two stimuli used in the control experiment.

\section{Results}

The frequencies of "away" and "toward" responses in each quadrant for each of the figures A and B were calculated for each subject and an analysis of variance for repeated measures was conducted on the "away" response frequencies. Neither the main effect for quadrant $(F=2.19$, df $=3,27)$, nor appendage $(F=$ $.008 . \mathrm{df}=1,9)$, nor the interaction was significant. A second analysis of the data using a four-quadrant sequence as the response unit showed that the frequencies of oscillation responses, rotation responses. and irregular responses were almost identical for the long appendage and the short appendage conditions.

\section{Conclusion}

Since the apparent motion of a single segment of the figure did not change significantly with the length of the vertical appendage, the function relating fragmentation to gap (Figure 3) cannot be accounted for by adding the independent apparent motions of the upper and lower components of the figures. Clearly, the whole is a great deal more than the sum of the parts in this case, and the degree of interaction between the parts depends on the size of the gap per se. Closure can now be operationally defined as apparent movement together in depth of all components of a tigure despite the presence of a gap; this movement must be greater than would be predicted from the joint probability of the individual motions of the parts.

\section{EXPERIMENT II}

The results of Experiment I immediately raise the following question. Is absolute or relative gap size on the retina the important determinant of closure? If the latter, then high-level processes would seem to be implicated, with the closure response perhaps depending upon the degree of similarity of the stimulus as a whole to some schema for a complete object. On the other hand. if the absolute gap is what is important, so that a given retinal gap is associated with the same amount of closure regardless of object size, more local processes involving topological representation of the stimulus at an early stage would seem to be responsible. For example, the interaction of line detector cells stimulated by the vertical lines on either side of the gap may play some role. Another hypothesis in line with an absolute gap determinant would be that "closure" depends upon the degree to which the entire gap and boundaries can be processed in a single glance. [See Hochberg (1968) on local depth cues and the processing of impossible figures.] This is not a highly probable explanation, however, since the visual angle size of the largest gap was only $1.3 \mathrm{deg}$.

In Experiment II, the above possibilities were explored by essentially repeating Experiment I with 12 new subjects, but with two observation distances instead of one. Order of the distances (140 and $280 \mathrm{~cm}$ ) was alternated from subject to subject. If relative gap size is the sole determinant, there should be no difference in the function relating closure to gap for the two distances, whereas if absolute gap size is the significant factor, the slope of the function should be halved at the greater distance.

\section{Results and Discussion}

The results are given in Figure 5 with least square fits to the data for the two distances. The slope of the dashed line is half the fitted slope for the $140-\mathrm{cm}$ condition. This would be the predicted value for the $280-\mathrm{cm}$ function were absolute retinal gap size the sole determinant of fragmentation. Since this predicted slope falls outside of the $95 \%$ confidence limits for the

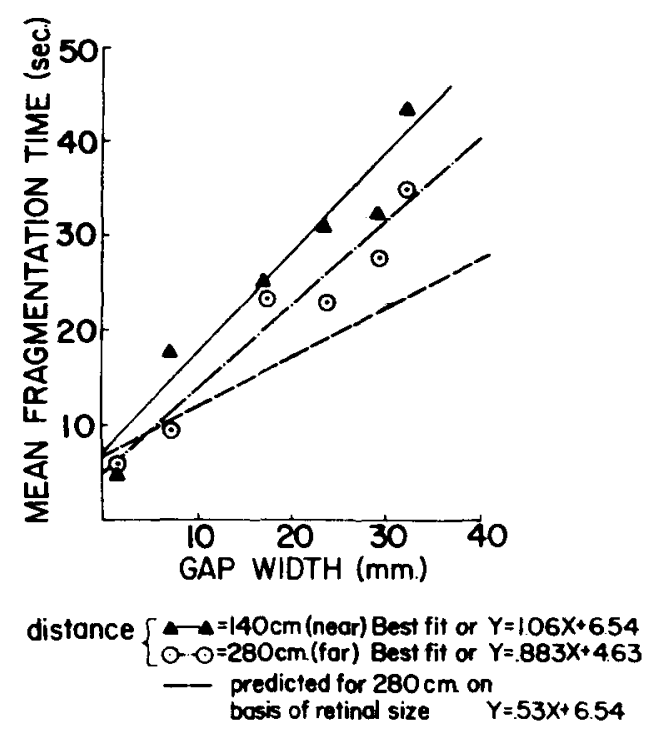

Figure 5. Fragmentation time as a function of gap for two observation distances. The dashed line represents predicted fragmentation for the farther distance based upon retinal gap size alone. 
slope of the empirical 280 -cm function, it seems that closure is not simply a function of absolute retinal gap size. More fragmentation occurred at the $280-\mathrm{cm}$ distance than expected solely on that basis. On the other hand. fragmentation time is not perfectly related to relative gap size either. An analysis of variance (repeated measures) of the data shown in Figure 5 revealed a highly significant $F$ ratio for distance $(\mathrm{F}=12.40 . \mathrm{dt}=1.11 . \mathrm{p}=.005)$ as well as for gap ( $F=9.23$, df $=5.11, p=.001)$. The interaction was not significant. This shows that identical contigurations do not demonstrate closure to the same degree if they differ in retinal size. There is more closure in the retinally smaller figure. It would clearly be desirable to vary retinal size independently of distance to separate constancy effects and retinal size effects. This was not done because of the difficulty involved in constructing very small figures by the present method. However, CRT displays will soon be available for this purpose.

\section{GENERAL DISCUSSION}

The present experiments have shown that there is an unambiguous sense in which closure occurs; depending on gap size, contours bounding a gap may be responded to as a unit when an ambiguous property, namely, movement in depth, is being resolved.

Closure is clearly not an all-or-none affair for simple figures. There is no critical gap size below which closure always occurs. In fact, it appears that any detectable gap will result in some fragmentation of a figure for some subjects.
Both topological and object-schema levels of processing appear to be involved in closure, even for a single gap. More than "proximity" grouping for collinear contours seems to be involved, although this may play a role. From the point of view of Gestalt psychology, the principle of "good continuation" is confounded with closure in the present experiments. Contour collinearity is one of a number of further parameters to be looked at in investigations of the nature of the processes underlying the present phenomenon and figure coherence in general.

\section{REFERENCES}

Bоввіт, J. M. An experimental study of closure as a threshold function. Journal of Experimental Psychology, 1942, 30. 273-294.

Gillam, B. J. Perceived common rotary motion of ambiguous stimuli as a criterion of perceptual grouping. Perception \& Psychophysics, 1972, 11. 99-101.

Heidgreder, E. Seven psychologies. New York and London: Century, 1933.

HochberG, J. E. In the mind's eye. In R. N. Haber (Ed.), Contemporary theory and research in visual perception. New York: Holt. Rinehart \& Winston, 1968. Pp. 309-331.

Holmes. D. S. Search for closure in a visually perceived pattern. Psychological Bulletin, 1968, 70, 296-312.

KofFKA, K. Principles of Gestalt psychology. New York: Harcourt Brace. 1935.

Schoenfeld, N. The metaphor of "closure." Psychological Review: 1941, 48, 487-497.

Street, R. F. A Gestalt completion test. Contributions to education. New York: Teachers College, Columbia University, 1931.

Wallach, H., \& O'Connell, D. N. The kinetic depth effect. Journal of Experimental Psychology, 1953, 65, 205-217.

(Received for publication December 16, 1974; revision received February 26, 1975.) 